\title{
Lean Transformation of Multinational Concerns
}

\author{
Rikke V. Matthiesen ${ }^{1}$, John Johansen ${ }^{1}$ \\ 1 Aalborg Universitet, Center for Industriel Produktion (CIP) \\ Fibigerstræde 16, 9220 Aalborg Ø, Denmark \\ WWW home page: http://www.cip.aau.dk/
}

\begin{abstract}
Competitive pressure demands that companies constantly strive to catch up to world class manufacturing performance and practice. Continuous change is a mode of competition for many companies [1]. This is a departure from the punctuated equilibrium model of change in which change is event triggered. This paper reports on exploratory studies from a multinational company adopting centrally managed pilot projects as a transformation mechanism for continuous change towards a lean business system and an organizational culture of continuous improvements (CI).
\end{abstract}

\section{Introduction}

Today companies may be in need of a swift transformation process. Here the global company faces the special challenge of transforming several sites simultaneously. Between such sites learning and knowledge sharing would be a means of obtaining an effective transformation process. This requires sameness in methods and tools and an effective means for this is centralized coordination of implementation.

In the literature one finds important discussions on the transformation process which overlap and supplement each other but do not match or subsume one another. In the first research on lean manufacturing [2] distinguished lean manufacturers by their performance regarding human effort, space, investment, and engineering hours. More recent work, e.g. [3, 4], uses constructs in terms of lists of practices to distinguish the lean operations. But recently there has been an increased focus on the missing correlation between working with lean practices and staying a healthy business. As opposed to practice lists and result orientation[5] and [6] emphasize a process oriented approach in building up process and continuous improvement capabilities respectively. [6] suggests doing improvements for the sake of improvements so as to build up quality awareness in the workforce. Building culture by such practicing of new ways of identifying and solving problems is in line with the view of organizational culture as learned responses to problems the organization

Please use the following format when citing this chapter:

Matthiessen, R.V. and Johansen, J., 2008, in IFIP International Federation for Information Processing, Volume 257, Lean Business Systems and Beyond, Tomasz Koch, ed.; (Boston: Springer), pp. 277-284. 
has encountered in the past [7]. [6, 8] emphasize the importance of policy deployment to direct continuous improvement efforts and numerous authors stress the importance of management attention and role modelling during transition periods.

\section{Approach}

The present research is based on experiences from a multinational company with more than 50 factories involved in a corporate-wide transformation.

The company is family owned and one Denmark's largest industrial companies with 18.000 employees globally. It is organized in three business units supplying components and control devices to OEM customers. The company has a financial goal for 2008 of reaching a turnover of $€ 3.200$ million from the current $€ 2.200$ million and of increasing its EBIT margin from 5,5\% to $10 \%$ so as to catch up with industry peers who are achieving $10 \%+$.

The company has a history of undertaking larger rationalization activities and has initiated efforts to develop and adopt its own lean business system. The programme was launched in 2003. The aim of the production programme is to cover all larger production areas with pilot projects in order to improve lead time and productivity in the short term and obtain a lean flow and a CI culture in the long term. Changes primarily take place on shop floor; they involve common lean tools and are lead by corporate change agents. The pilot projects should work as training grounds for local project members and management who are responsible for the continued sustaining, evolving, and cascading of implemented changes so as to drive a transformation of the entire production organization.

The programme is organized with two senior consultants who are in charge of its execution. They also consult factories on tactical issues regarding their efforts to become lean. Corporate change agents are mainly recruited from within the company and are expected to stay with the programme for 2-3 years. The pilot projects follow a standardised schedule but the focus of each project is adapted to the particular business needs in the area. Between these pilot projects, the corporate project leaders exchange experiences and tools. So far this sharing has resulted in decisions regarding demands for management support, mandatory tools implementation and project management elements, awareness of stress symptoms, teambuilding events, and greater emphasis on high productivity targets and layout changes.

\subsection{Research Method}

The aim of this study is to identify key themes and hypotheses relevant to the transformation process initiated through pilot projects, driven by a corporate staff of experts. An inductive and exploratory qualitative approach was adopted as a research approach. The focus area is twofold: 1) Management reflections, plans and actions regarding a transformation, 2) The dynamics arising during and after pilot projects.

Interviews are chosen as the primary data. As a starting point for the interviews, a list of themes relevant to a transformation process was developed from researchers 
in the field of organizational change. During the interviews probing questions were asked based on observations of shop floor practices as well as experiences from one of the researchers $1 \frac{1}{2}$ years of employment within the change programme.

\subsection{Case Descriptions}

Four factories have been selected for this initial exploratory research. They are all located in Denmark, and three of them are part of the same division. Factories vary in size and number of shifts, see Table 1, but their processes are quite similar. Three of the four factories are organized with operational team managers; factory beta stands out as supervisors in this factory report directly to the plant manager.

Table 1. Comparison of factories

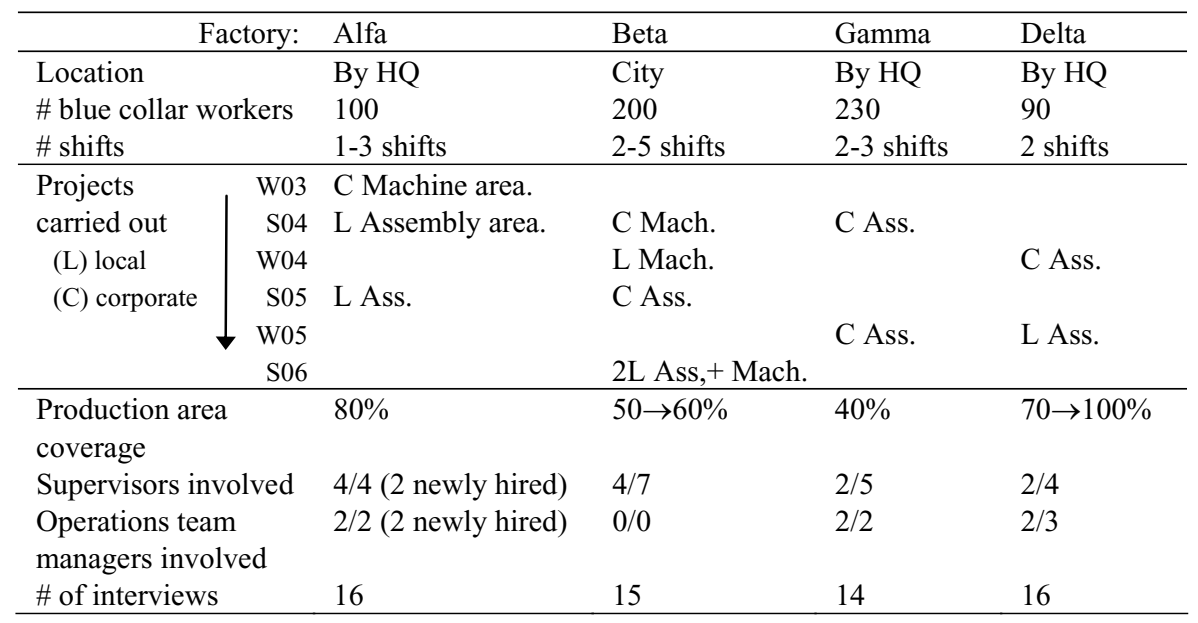

All four factories have or have had change agent(s) employed with direct report to the plant manager. These change agents participated as team member in the centrally managed pilot projects in the respective factories. In factories alfa, beta, and delta, the change agents had subsequently been responsible for locally managed project(s) more or less copying the centrally managed project. A factor common for the locally managed projects though was that they resulted in less significant changes and they spent more time on involving employees and utilizing their ideas as a means of reducing resistance and increasing buy in.

The factories have been subject to corporate projects in different stages of the roll out and have despite similarities also taken different routes from there. Factory alfa and beta have in addition to pilot projects been focusing on broader issues such as flow across the plant. Both factories experienced projects resulting in employee and supervisor resistance or dissatisfaction, and in both factories plant managers had been quite involved in discussing the future state picture. It appeared that they saw an improved flow as the means for convincing employees, winning them over, and changing behaviour and mindset. Factory delta focused on projects covering whole 
value streams one by one. Projects would be used as a platform for improving further towards a more cost efficient and lean operation. In factory gamma the organization had been swamped in ramping up a new production. Therefore focus on lean had been reduced during a period following the first corporate project. Now, however, the company is working with team building and employee mindset which is seen as the platform to build better performance on.

Comparing these three tactics, factory delta stands out as not focusing so intensely on mindset changes but primarily on improving performance and thus having a very clear vision of the future state. Several factors could help explain this. The factory had experienced less frustrating projects, and has fewer shifts and more middle managers. The organization also has a good track record of increasing performance and a general sense of sufficiency in CI capabilities.

All interviews from the beta case have been transcribed. These transcriptions along with impressions from the other cases were used to form the initial analyses frameworks. Seventy percent of interviews conducted in cases alfa, gamma, and delta have been reviewed in order to test and expand the frameworks.

\section{Analysis}

\subsection{Six Expressed Opinions about Change Goals}

The interviews showed that the organizations were not engaged in a broad practicing of the new tools outside the pilot area as a means of reaping improvement potentials, building up capabilities, and increasing improvement awareness across the organization. The most obvious transformation mechanism triggered by the pilot projects was that of cascading change by copying the pilot project approach locally. But a CI culture is not built up during a pilot project. Building a culture requires that the implemented systems are used as a platform for ongoing experimentation with new behaviours $[5,6]$. In regard to using pilot projects as such platforms, the interviews left the impression though, that only few interviewees considered the organization to be in the middle of a transformation and that many only focused on equipment problems in relation to CI. This signifies that the projects build up only a low transformation drive towards a new culture and it points to the relevance of investigating this transformation drive and the mechanisms affecting it.

This section presents 6 categories of statements about change and the future state found in the interviews. From these categories a framework of transformation drive is sketched out. This is applied in an analysis of the levels of the transformation drive within various organizational functions. From this the main trends for each function are identified and deviations are analysed.

Interviews were scrutinized for statements about the changes, about future state perspectives, and about opportunities for improvement. It appeared that 6 groupings were necessary to distinguish between the most significant differences. In the following each of these 6 groupings are described.

1. Project thinking: Interviewees expressed a view that other things were on the agenda now. They did not express that the project was a step on a journey or 
anticipate that they would be responsible for or participate in significant changes except for maybe copying the project to other areas.

2. Transformation: Interviewees expressed that they had specific and multiple goals regarding culture, capabilities and performance and they were actively trying to achieve those. [9] quotes authors for seeing transformative change as episodic and abrupt, but in this case the opposite is found to apply as managers need to show that changes have not finished together with the project.

3. Slave of the system: Interviewees talked about the surrounding system as limiting their internal performance. In the following such views are listed according to declining degree of strength: Actively opposing implemented tools, seeing more resources as only means for solving problems, denying opportunities to improve should exist within the system, being passive or helpless, expressing that change only happens occasionally but generally the best we can do is to do our best.

4. Mastering the system: As opposed to (3) some interviewees saw the technical system from outside in. They expressed the view that the rate at which we usually tackle our technical problems in the system is sufficient.

5. Changing people: Some interviewees saw employees as part of the system. They were slowly pushing so as to develop some mindset or behaviour aspect. In a less active version they were hoping for a better behaviour among colleagues.

6. Desiring a more involving and lively process: Some interviewees expressed that the change process should get higher focus. They were wishing for more dialogue and involvement so as to shape the future state together in a more creative process bringing more topics into the debate.

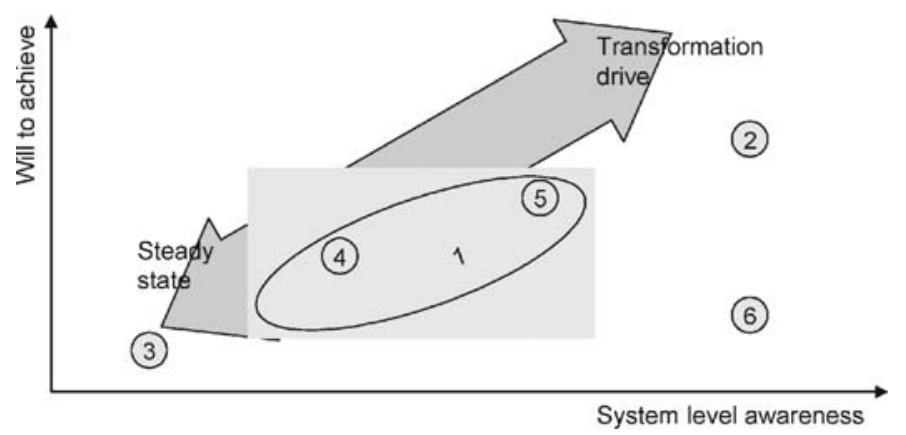

Fig. 1. A tentative transformation drive framework of future state perspectives

Each grouping expresses some desire for progress but their perceived support of the transformation varies. Two dimensions distinguishing the underlying values were tentatively chosen to form a framework to range the groupings according to the transformation drive they expressed: One dimension relates to desire for speed of change, willingness to work for change, and ability to make it happen which is categorized as 'Will to achieve change'. The other dimension is named 'System level awareness' and refers to Boulding's 1956 classification and the systems perspective taken: What constitutes the system to improve, and what capacity does the system contain for action. Applying a higher level systems perspective aids in identifying 
improvement levers effectively and constructively. Using these dimensions the six groupings of statements can be arranged according to their transformation drive as displayed in Fig. 1. The axes may not be entirely independent but the model should illustrate that moving from the bottom left to the top right represents an increasing transformation drive.

\subsection{Trends and Deviations Related to Organizational Functions}

The individual interviews were analysed according to this framework of transformation drive. To some extent views within organizational functions or levels were alike - more so than opinions expressed within the same factory or project. Common education, work experience, and job requirements build occupational cultures [10] but also the individual project role, e.g. a supervisor would have, would be similar across projects. Still there were deviations from the main trend within each organizational function or level. Analysing these deviations triggered some insights into the mechanisms that affect transformation drive in relation to the pilot projects. In the following arrows $(\leftarrow \uparrow \rightarrow \downarrow)$ are used to indicate the directions interviewees appear to have moved in the transformation drive framework.

Supervisors: Most of the supervisors viewed the project as a project (1) and many took on the slow development view (5) with increased focus on cross training $\rightarrow$. Only three supervisors accounted for a transformation process - two of these in past tense. These two had both been involved in projects that had built up frustration among employees and they therefore had been deeply involved in changing mindsets, motivating people and bringing back productivity levels. The third account was from a recently finished project that had left a lot of details for the local organization. Judging from the two accounts in past tense this increased engagement throttles down as motivation comes back to normal and as the implemented systems become routine and enables the production teams to handle smaller coordination tasks on their own $\uparrow \downarrow$. A couple of supervisors had overtaken their position after the pilot projects had finished; they either directly opposed the implemented systems or did not see the benefit in all of them. It therefore appears that education is required for new supervisors to understand and benefit from the implemented systems $\leftarrow$.

Operators and team coordinators: Most of the operators and team coordinators viewed the project as a one time event (1). Some operators expressed a passive attitude not really seeing any potential to improve either operations or their conditions as operators (3). This was especially so on assembly lines where operators had gotten a considerable larger workload as a result of the project. In these assembly areas operators felt that interesting parts of their jobs had been taken away and that they now worked more like robots while team coordinators were given all the tasks that had previously been a welcome break or a challenge to deal with $\downarrow \downarrow$. Especially in areas where the team coordinator had a strong coordinating role, some team coordinators were hoping for their operator colleagues to take more responsibility $(5) \rightarrow \uparrow$. In the two machining areas, team coordinators had been appointed reluctantly. They gave examples of how they on some aspects opposed the implemented systems as a means of insisting on not coordinating their own colleagues $\leftarrow$. Some operators and team coordinators had seen the project as a step 
towards a different culture and were hoping for a more lively approach to improvements and learning (6) - some wanted more time or latitude from management and others were hoping for their colleagues to take a greater interest in learning and developing the area. Interest in a more lively development seems to be very individual, but in general a positive experience with prior involvement in some improvement activities appeared to underlie this desire $\rightarrow$ while group dynamics appeared to affect it negatively. The issues described above signify a potential for utilizing operator and team coordinator viewpoints for improvements of the system.

Support functions: Most support personnel expressed project thinking (1). Some engineers were involved in the daily operations utilizing the new systems but the projects they worked on did not relate to lean or to the productivity improvement project as such. Planners were generally absorbed with daily operations and thus had little opportunity to work with lean tools. However, exceptions in both directions were found. It appeared that participation in the projects did bring about increased 'System level awareness' $\rightarrow$. While operations personnel that had not been involved in the projects tended to have their tasks staying on the task lists for too long and thus affected transformation drive negatively in the entire pilot organisation $\downarrow$.

Change agents: They expressed the transformation view (2) - more than project participants involved with daily operations $\rightarrow \uparrow$. They were quite involved with discussions about the future state and seemed to be important sparring partners for supervisors and plant managers.

Managers: Plant managers were all aiming at a transformation (6), and the transformation appeared to take up a relatively large part of their agenda $\rightarrow \uparrow$. Two newly hired operations team managers from external companies expressed a desire for a more energetic change process. Only two operations team managers expressed the project view (1). They were both worked in areas that had been substantially transformed during projects and now performed at a new level. The implemented systems formed a solid platform to maintain this level and the managers expressed greater confidence in the organizations capacity to accomplish improvements $\uparrow$.

Overall primarily change agents, managers, and some team coordinators appear to be moving towards a larger transformation drive. With supervisors and support personnel, the movement in the framework is primarily horizontal towards increased systems awareness. But in some cases especially with operators, movements are in the direction away from transformation drive.

\section{Summary}

The pilot project organization, form and content is successful in leveraging performance and practices. Though as a transformation mechanism the approach is not unproblematic. The pilot projects favour management, change agents and to some degree the new team coordinator role. Post pilot transformation focus is on copying projects as a means of building new behaviours via the copied systems and on building project management skills. It is not evident that this is a strong transformation mechanism since corporate managed projects already have demonstrated that as a platform for learning and developing a new culture the pilot project is not a strong concept. Two years after a pilot project, focus is still limited to 
technical problems. And the pilot projects have built up only a temporary transformation drive among supervisors on the one hand while they have built up resistance or passivity among operators on the other hand. This group is generally not activated in the development of a new culture and where they potentially could be, this has not been supported and made use of. Comparing organizations, it is found that existing organizational culture has a strong influence on pilot project outcomes. But where locally managed projects have adjusted to better fit local organizational culture, dynamics within the corporate programme have increased the focus on mechanistic efficiency. This is problematic since an effective corporate organization is allowed a stronger position and thus maintains the focus on projects as the primary transformation mechanism.

\section{References}

1. S.L. Brown and K.M. Eisenhardt, The Art of Continuous Change: Linking Complexity Theory and Time-paced Evolution in Relentlessly Shifting Organizations, Administrative Science Quarterly 42, 1-34 (1997).

2. J.P. Womack, D.T. Jones, and D. Roos, The Machine that Changed the World (Harper Perennial, 1990)

3. C. Karlsson and P. Åhlström, Assessing changes towards lean production, International Journal of Operations and Production Management 16(2), 24-41 (1996).

4. R. Shah and P.T. Ward, Lean manufacturing: context, practice bundles, and performance, Journal of Operations Management 21, 129-149 (2003).

5. S. Spear and H.K. Bowen, Decoding the DNA of the Toyota Production System, Harvard Business Review 77(5), 97-106 (1999).

6. M. Imai, Kaizen (The KAIZEN Institute Ltd., Skive, 1986)

7. E.H. Schein, Coming to a New Awareness of Organizational Culture, Sloan Management Review 25(2), 3-16 (1984).

8. J. Bessant and S. Caffyn, High Involvement innovation through continuous improvement, International Journal of Technology Management 14(1), 7-28 (1997).

9. D.C. Dunphy and D.A. Stace, Transformational and Coercive Strategies for Planned Organizational Change: Beyond the O.D. Model, Organizational Studies 9(3), 317-334 (1988).

10. E.H. Schein, Three Cultures of Management: The Key to Organizational Learning, Sloan Management Review 38(1), 9-20 (1996). 